Original Article

\title{
Ten Years' Experience in Surgical Treatment of Right Middle Lobe Syndrome
}

\author{
Saviz Pejhan, MD,${ }^{1}$ Farshid Salehi, MD, ${ }^{1}$ Shanay Niusha, MD, ${ }^{1}$ \\ Behrooz Farzanegan, MD, ${ }^{2}$ and Kambiz Sheikhy, $\mathrm{MD}^{3}$
}

\begin{abstract}
Purpose: In this study we present the clinical, radiological, pathological, bronchoscopic and surgical results of $\mathbf{4 0}$ patients with diagnosis of middle lobe syndrome who were referred to our thoracic surgery unit for surgical intervention in a 10 years period.

Methods: Forty patients with obstructive and non-obstructive causes of middle lobe syndrome referred to our thoracic surgery unit. Clinical data were collected from the patients' records in a ten years period. This study evaluates diagnostic approaches and surgical treatments in right middle lobe syndrome.

Results: We studied 23 females $(57.5 \%)$ and 17 males $(42.5 \%)$ with a mean age of 31.7. Clinical findings were cough $95 \%$, sputum $80 \%$ and intermittent hemoptysis in $50 \%$ of patients. Middle lobe collapse was seen in CT scan of all patients. Bronchiectasis was the most common pathologic finding (55\%). Tuberculosis was not rare and was final pathology in $20 \%$ of patients. In three patients ruptured hydatid cyst was final finding. Surgery was done without mortality and with only minor complications.

Conclusion: Lobectomy of right middle lobe is a good therapeutic option in these patients. Due to high prevalence of tuberculosis and hydatid cyst in Middle Eastern countries these two must be considered as causes of middle lobe syndrome.
\end{abstract}

Keywords: Middle Lobe Syndrome, Bronchiectasis, Hydatid Cyst, Tuberculosis, Surgery

\section{Introduction}

Right middle lobe syndrome (RML) is a bronchoconstrictive and uncommon disorder of the lung. The

${ }^{1}$ Tracheal Diseases Research Center, National Research institute of Tuberculosis and Lung Diseases (NRITLD), Shahid Beheshti University of Medical Sciences, Tehran, Iran.

${ }^{2}$ Telemedicine Research Center, National Research institute of Tuberculosis and Lung Diseases (NRITLD), Shahid Beheshti University of Medical Sciences, Tehran, Iran.

${ }^{3}$ Lung Transplantation Research Center, National Research institute of Tuberculosis and Lung Diseases (NRITLD), Shahid Beheshti University of Medical Sciences, Tehran, Iran.

Received: October 5, 2014; Accepted: December 9, 2014 Corresponding author: Kambiz Sheikhy, MD. Lung Transplantation Research Center, National Research institute of Tuberculosis and Lung Diseases (NRITLD), Shahid Bahonar Ave, Daar-abad, Tehran 19569-44413, Iran

Email: ksheikhy@gmail.com

(C)2015 The Editorial Committee of Annals of Thoracic and Cardiovascular Surgery. All rights reserved. term "right middle lobe syndrome" was originally coined in 1948 by Graham, et al. ${ }^{1)}$ as middle lobe atelectasis due to bronchial compression caused by mediastinal lymph node enlargement. Since that report, many other investigations have been reported which have led to the change in definition of middle lobe syndrome. Since 1966, term of middle lobe syndrome refers to all middle lobe atelectasis and collapse of right middle lobe even when the bronchial compression is not present. Pathophysiologically, there are two forms of middle lobe syndrome: obstructive and non-obstructive. This syndrome is characterized by chronic cough with sputum, episodes of hemoptysis and repeated chronic pneumonitis. ${ }^{2)}$

Treatment of middle lobe syndrom (MLS) is dependent on the underlying cause. Patients with non-obstructive MLS usually respond to medical therapy. If conservative treatment fails or for obstructive cases, surgical removal will be an option. . $^{3,45}$ 
There is limited knowledge on symptoms, histopathology and benefits of surgery in patients with middle lobe syndrome. Considering that in many of previous studies case groups were small or articles were case reports, with regard to significant number of patients (40 patients) who underwent surgery in our center during 10 years period we decided to provide our experiences on clinical, radiological and pathological findings and results of surgical treatment in our patients.

\section{Patients and Methods}

This descriptive, retrospective existing-data study was performed on 40 patients with right middle lobe syndrome who had undergone surgery in our center in a 10 years period between 2000-2010. The variables were: age, sex, signs and symptoms, duration of symptoms, history of admission due to pneumonia or hemoptysis, paraclinical findings, bronchoscopic findings, type of surgery, complications and pathological findings. All of these patients have been referred for surgery due to diagnosis of obstructive MLS or failure of medical treatment in non-obstructive MLS. Radiological evaluation with chest computed tomography (CT) scan was done in all patients.

On all patients, rigid or fiberoptic bronchoscopy was performed before surgery. The double-lumen endotracheal tube was used in all patients except two children. A posterolateral thoracotomy through the fifth intercostals space was performed. Visceral and parietal pleural adhesions were found in the majority of patients. It was most severe in patients with tuberculosis. These adhesions were particularly problematic in cases with bronchiectasis. Then the main fissure was exposed, and the right middle lobe arteries were ligated. In the next step, middle lobe vein was ligated, and the bronchus was cut and sewn. Due to multiple enlarged calcified lymph nodes, dissection around right middle lobe arteries was difficult and careful attention was needed.

Except for three patients with carcinoid tumor, lobectomy of middle lobe was performed in all patients. On one of those three patients, bronchotomy and tumor local resection was performed. On one other, lower and middle bilobectomy, and on third patient upper and middle sleeve lobectomy was performed. All patients were followed between 2-12 months. SPSS software (version 16) was used to input and analyze data.

\section{Results}

Patients included 23 females and 17 males with a mean age of 31.7 years. Common symptoms were as follows: cough $95 \%$, sputum $80 \%$, intermittent hemoptysis $50 \%$, thoracic pain $25 \%$, massive hemoptysis $17.5 \%$. Duration of symptoms from onset till surgical treatment was $1-5$ years in $25 \%, 5-8$ years in $40 \%$ and in six patients more than 8 years (8-22 years). All the patients had the history of recurrent pneumonia and purulent sputum, and most of them had been under medical treatment frequently due to a lung infection. Two of the patients had been treated with anti-tuberculosis drugs but just one of them was infected by tuberculosis at the final diagnosis. Radiological study was done by spiral $\mathrm{CT}$ in all patients that revealed partial or complete atelectasis of RML in all patients. Additional findings in CT scan are shown in Fig. 1.

Pulmonary function tests had been performed on 20 patients based on the records, and except for three patients who had FEV1 and FVC less than 50\% of predicted value, others' test results were normal. In three cases who had abnormal pulmonary tests, two patients had the history of cough and sputum and recurrent pulmonary infections for more than 10 years, and one case had already undergone right lower lobectomy due to bronchiectasis. PPD test in all patients were negative. Bronchoscopic findings are shown in Fig. 2.

There was not any mortality or major complication during the operation and postoperative period. Minor postoperative complications included lung collapse after chest tube removal in one patient and prolonged air leakage in another. In one patient, the lung did not expand after inserting chest tube so we had to remove it and insert into new place. All of these three patients recovered. All patients were followed up for 2 months to one year. All the patients survived at the end of study time and did not have clinical symtoms. Patients with confirmed TB in their pathology findings went under anti-tuberculosis treatments. Patients' pathology findings are shown in Fig. 3.

\section{Discussion}

Right middle lobe syndrome is a spectrum of diseases from recurrent atelectasis and pneumonitis to bronchiectasis of the middle lobe. ${ }^{4)}$ In this study, we tried to analyze the symptoms, radiographic and histo- pathological features and result of surgical treatment of patients who underwent lobectomy for middle lobe syndrome over a 10-year 


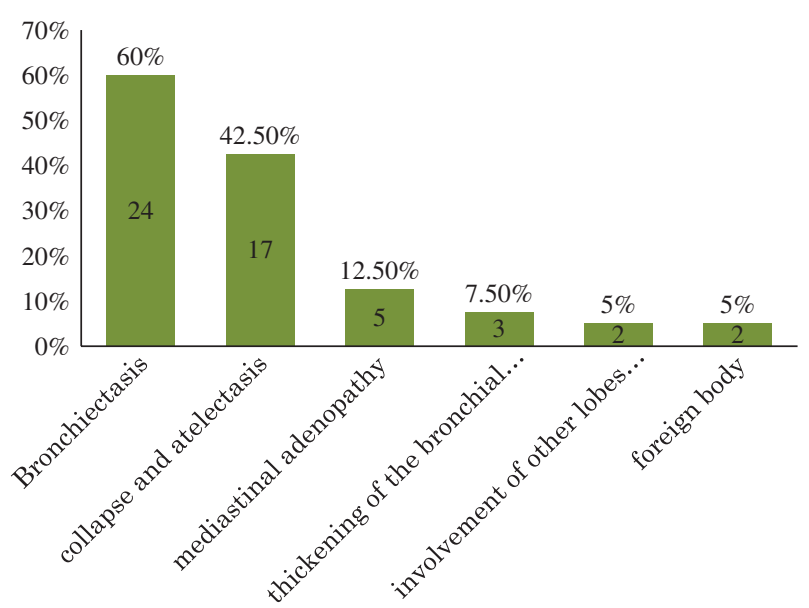

Fig. 1 Computed tomography (CT) scan findings in 40 patients with middle lobe syndrome.

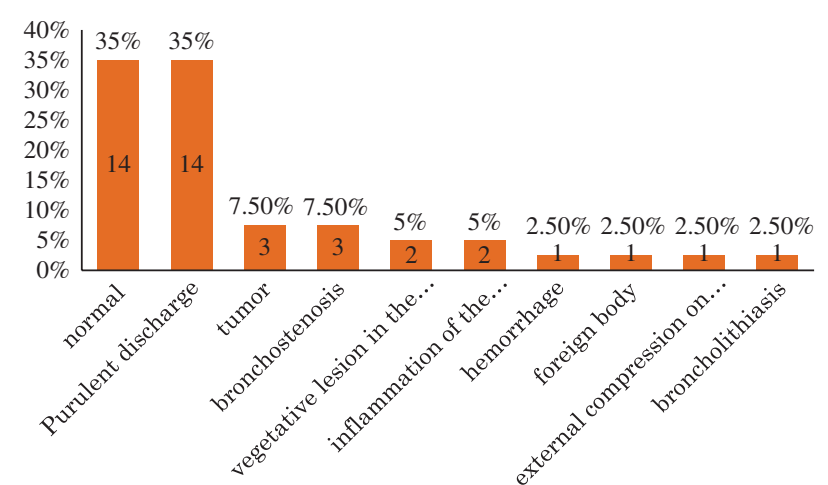

Fig. 2 Bronchoscopic findings in 40 patients with middle lobe syndrome.

period. Diagnosis of this syndrome is based on patient's history and findings in bronchoscopy and radiologic imaging. Most patients experience intermittent attacks of respiratory infections, recurrent bloody sputum, and right middle lobe atelectasis. The clinical symptoms that our patients had were cough, sputum, hemoptysis, chest pain and history of treatment for recurrent pneumonia, a similar finding to most other studies. ${ }^{6,7,8)}$

We found that our patients were younger than cases in Kwon ${ }^{9)}$ study (with the mean age of 47 years) and Einarsson's ${ }^{7)}$ cases (mean age of 55.7 years), but older than patients of Meterog $\mathrm{lu}^{10)}$ study with the mean age of 12.3 years.

Like some other studies, in our study middle lobe syndrome was more common in females. In the study by Kwon, et al., $71 \%$ of patients were females. In the study by Einarsson 15 out of 18 patients were women.

Currently the modality of choice for diagnosis of MLS is chest CT scans. ${ }^{8)}$ Plain chest radiographies may

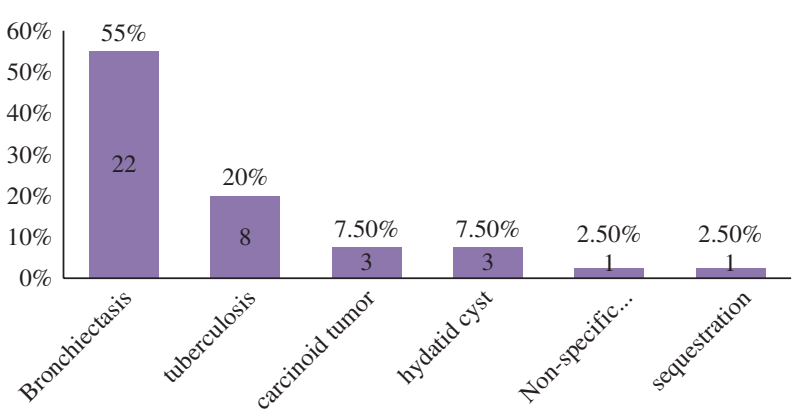

Fig. 3 Pathologic findings in 40 patients with middle lobe syndrome.

show broncho stenosis, right middle lobe collapse, inflammatory changes, and bronchiectasis. The radiographic findings most often seen in our study were atelectasis, collapse, and bronchiectasis which the last one were more than the rate of previous studies, and our broncho stenosis cases were less. ${ }^{4,9)}$ This is in agreement with the study by Sehitogullari, et al. ${ }^{11)}$ in that study HRCT revealed bronchiectasis in $11(55 \%)$ patients, atelectasis and bronchiectasis in five (25\%) patients, and destroyed middle lobe in four (20\%) patients. Imaging findings in Einarsson's study were atelectasis in nine patients (50\%), consolidation in nine $(50 \%)$, bronchiectasis in seven (39\%) and patchy infiltrates in four (23\%).

The etiology of atelectasis of middle lobe in nonobstructive cases is not completely understood. Certain anatomical characteristics make the middle lobe susceptible to obstruction if inflammation or infection occurs. The narrow diameter of the middle lobe bronchus and the acute take-off angle besides the relative anatomical isolation of the middle lobe create poor conditions for drainage. ${ }^{78)}$ Also, it was previously reported that swelling of lymph nodes in response to either acute or chronic inflammation can narrow the bronchus and cause atelectasis, chronic bronchitis, or bronchiectasis which can gradually lead to collapse and narrowing of the bronchial divisions. In addition, the middle lobe contains only scanty paranchymal bridges due to the deep fissures that isolate this lobe which provides barriers to collateral ventilation. This inefficient collateral ventilation of the middle lobe, especially in patients with complete fissures, reduces the chance of reinflation once atelectasis has occurred and can cause obstruction. Thus in these patients, in spite of collapse and clinical signs, no obstruction of the middle lobe bronchus was seen during bronchoscopy. In earlier studies the more common bronchoscopic findings were obstructive ones. ${ }^{1)}$ In a study by Wagner ${ }^{4)}$ bronchoscopic findings showed that in 38\% of 
these patients there was a kind of obstruction or tumor, in $17 \%$ nonspecific findings, and in $45 \%$ of the patients' bronchoscopy was normal. But the more recent studies ${ }^{10,11,12)}$ show non-obstructive reasons as the most common causes. Our normal bronchoscopies were same as previous studies, but we had more nonspecific findings such as infectious discharge in the right middle lobe bronchus, inflammation or granulation tissue, and some more specific findings includes bronchostenosis, tumor or foreign body.

Wagner, et al. ${ }^{4)}$ in 1983 in a review article, of 933 cases have ever reported, stated the following pathologic findings of right middle lobe syndrome: inflammation $47 \%$, bronchiectasis $15 \%$, malignant tumors $22 \%$, tuberculosis $9 \%$, benign tumors $2 \%$, aspiration $2 \%$, miscellaneous $3 \%$. Statistics comes from our patients indicate that tumors as a reason in our patients were less than other studies and the most common pathologic finding among our patients was bronchiectasis. This is in agreement with the study by Einarsson, et al. ${ }^{7}$ and Kwon, et al. ${ }^{9)}$ and Eskenasy ${ }^{13)}$ in which the bronchiectasis was the primary finding. But in our study, the tuberculosis as the reason of MLS in comparison was more common than previous studies.

Of patients with tuberculosis, bronchoscopy revealed bronchostenosis in one of 8 cases and hemorrhagic inflammation in another patient. Only in 2 patients tuberculosis was confirmed before surgery and both of them had vegetative lesions in the orifice of middle lobe bronchus in their bronchoscopy, and their TB was confirmed by biopsy. Interestingly, in all TB cases, PPD test, sputum smear and culture and BAL were negative for BK.

Pathologic findings show that the cause of MLS in 3 of our patients was hydatid cyst. In reviewing references we could not find any study mentioned hydatid cyst as one of pathologic findings in the middle lobe syndrome. None of these cases had any history of brackish sputum or laminated membrane excretion and the diagnosis was confirmed by pathologic findings after surgery. One of these patients had been under antituberculosis treatments due to prolonged cough and hemoptysis and hemithorax pain for 2 years, and in his chest $\mathrm{x}$-ray and CT scan calcification in the hilar lymph nodes and parenchyma of lung was seen.

Treatment of MLS is directed at the underlying cause. There are no good recommendations available about appropriate time of surgery. Patients who have repeated episodes of infection with lobar collapse and evidence of broncho stenosis, tumor, or obstruction from enlarged lymph nodes are best treated by surgical resection. Surgery may also be indicated if medical management fails and symptoms persist with chronic changes found on radiological imaging studies such as scarring, fibrosis, or abscess formation. Foreign objects can often be removed with flexible fiberoptic or rigid bronchoscopy. If no endobronchial obstruction is present, the patient can be treated medically. ${ }^{5,6,11)}$

Enlarged lymph nodes around the middle lobe bronchus were present in our patients and made the operation more complicated. In spite of this difficulty, the post-operative complications were rare and we did not have any mortality. Significant percentage of our patients was diagnosed with tuberculosis although pre-operative evaluations including PPD, BAL, and sputum smear were negative, and four of patients had normal bronchoscopic findings. Besides, the cause of MLS in 3 of our patients was hydatid cyst that was not mentioned in any previous studies.

Previous studies agree with surgical treatment on patients with recurrent infection attacks with lobar collapse and evidences of bronchostenosis, tumor or obstruction. However, regarding this fact that tuberculosis and hydatid cyst are endemic in our country, if we try to choose medical treatment strategy just bases on clinical symptoms or imaging findings, we may miss some patients with tuberculosis or ruptured hydatid cyst and lead them to serious complications.

We recommend that, despite of great difficulty in right middle lobectomy, surgical intervention could be a good option for the patients with persistent atelectasis of right middle lobe with no response to medical therapy and patients with no preoperative diagnostic findings.

\section{Acknowledgment}

None declared.

\section{Funding}

There was not any funding.

\section{Conflicts of interest}

None declared

\section{References}

1) Graham E, Burford T, Mayer J. Middle lobe syndrome. Postgrad Med. 1948 Jul; 4: 29-34.

2) Joseph I. Miller Jr. In Shields TW, ed. General Thoracic Surgery 7th ed. Philadelphia.Wolters KluwerLippincott Williams \& Wilkins, 2009: 1117-1129. 
3) Gudmundsson G, Gross TJ. Middle lobe syndrome. Am Fam Physician 1996; 53: 2547-50.

4) Wagner RB, Johnston MR. Middle lobe syndrome. Ann Thorac Surg. 1983; 35: 679-86.

5) Ayed AK. Resection of the right middle lobe and lingula in children for middle lobe/lingula syndrome. Chest 2004; 125: 38-42.

6) De Boeck K, Willems T, Van Gysel D, et al. Outcome after right middle lobe syndrome. Chest 1995; 108: 150-2.

7) Einarsson JT, Einarsson JG, Iasksson H, et al.: Middle lobe syndrome: a nationwide study on clinicopathological features and surgical treatment. ClinRespir J 2009; 3: 77-81.

8) Gudbjartsson T, Gudmundsson G. Middle lobe syndrome: a review of clinicopathological features, diagnosis and treatment. Respiration 2012; 84: 80-6.
9) Kwon KY, Myers JL, Swensen SJ, et al. Middle lobe syndrome: a clinicopathological study of 21 patients. HumPathol. 1995; 26: 302-7.

10) Metero glu F, Sahin A, SevvalEren T. Middle lobe syndrome: a retrospective analysis. Türk Göğüs Kalp Damar Cerrahisi Dergisi 2013; 21: 718-722.

11) Sehitogullari A, Sayir F, Cobanoglu U, et al. Surgical treatment of right middle lobe syndrome in children. Ann Thorac Med 2012; 7: 8-11.

12) Saha SP, Mayo P, Long GA, et al.: Middle lobe syndrome: diagnosis and management. Ann ThoracSurg 1982; 33: 28-31.

13) Eskenasy A, Eană-Iorgulescu L. Pathology of the middle lobe syndromes. A histopathological and pathogenetic analysis of sixty surgically-cured cases. Med Interne 1982; 20: 73-80. 